Pacific Journal of Mathematics

FOURIER SERIES WITH BOUNDED CONVOLUTION POWERS 


\title{
FOURIER SERIES WITH BOUNDED CONVOLUTION POWERS
}

\author{
Charles H. Heiberg
}

Let $f$ be defined on $T^{n}$ and have an absolutely convergent Fourier series

$$
f\left(e^{i \sigma}\right)=\boldsymbol{\Sigma} f_{m} e^{i m \cdot \sigma} .
$$

Set $\|f\|=\Sigma\left|f_{m}\right|$. In this paper sufficient conditions for $\left\|f^{k}\right\|=$ $0(1)$, as $k \rightarrow \infty$, are obtained.

THEOREM. Let $f$ be defined on $T^{n}$, have an absolutely convergent Fourier series and satisfy

$$
\left|f\left(e^{i \sigma}\right)\right| \leqq 1 \text { for all } \sigma \text {. }
$$

If for each $\sigma$ such that $\left|f\left(e^{i \sigma}\right)\right|=1$ there exists a rotation $\lambda$ of $R^{n}$, a polynomial $\rho$ such that $\operatorname{Re} \rho(\tau)>0$ for all $\tau \neq \overrightarrow{0}$, an n-tuple $p$ of positive integers such that $\rho\left(\left\langle r^{1 / p} \tau_{i}\right\rangle\right)=r \rho(\tau)$ for all $r>0$, and a function $\gamma$ in $C^{m}\left(R^{n}\right), m=\max \left(n+1, p_{1}, p_{2}, \cdots, p_{n}\right)$, such that

$$
\gamma(\tau)=o\left(\sum_{i=1}^{n} \tau_{i}^{p_{i}}\right), \quad \tau \longrightarrow \overrightarrow{0},
$$

and if for all $\tau$ in some $R^{n}$-neighborhood of $\overrightarrow{0}$

$$
f\left(e^{i(\sigma+\lambda(\tau))}\right)=c \exp (\beta \cdot \tau i-(\rho+\gamma)(\tau)),
$$

where $|c|=1, \beta \in R^{n}$, then

$$
\left\|f^{k}\right\|=0(1), \text { as } k \longrightarrow \infty \text {. }
$$

It is shown in $\S 2$ that this theorem extends a result obtained by B. M. Schreiber in 1970 .

1. Introduction. Let $B$ and $D$ denote the open and closed unit discs respectively. The problem of characterizing those functions $f$ of $n$ complex variables which are analytic on $D^{n}$ and for which (4) holds has been solved only for $n=1$. See [1, 2]. For the general case, $n$ arbitrary, sufficient conditions on $f$ and necessary conditions on $f$ have been given $[9,6]$ and related problems have been studied $[4,5]$. Unsolved, even in the case $n=1$, is the problem of determining all functions analytic on $B^{n}$ for which (4) holds. This problem is equivalent to the problem of determining all endomorphisms of the Banach algebra of power series of $n$ complex 
variables which converge absolutely on $T^{n}$. See $[8,7]$.

2. Remarks, definitions and notation. To see that the theorem in this paper extends Schreiber's result, Theorem 7.5 in [9], let $n=2$ and consider $f(z)=h(z) g\left(z_{1}\right) 1\left(z_{2}\right)$ where

$$
\begin{aligned}
& h(z)=z_{1}^{2} z_{2}^{2}-\left(\frac{z_{1}-1}{2}\right)^{4}\left(\frac{z_{2}-1}{2}\right)^{4}, \\
& g\left(z_{1}\right)=z_{1}^{3}+\left(\frac{z_{1}-1}{2}\right)^{6}, \\
& 1\left(z_{2}\right)=z_{2}^{6}+\left(\frac{z_{2}-1}{2}\right)^{12} .
\end{aligned}
$$

The function $f\left(e^{i \tau}\right)$ has one absolute maximum, namely at $\tau=$ $(0,0)$, and about this maximum has a local expansion of the form

$$
f\left(e^{i \tau}\right)=c \exp \left(\beta \cdot \tau i-a \tau_{1}^{6}-b \tau_{1}^{4} \tau_{2}^{4}-d \tau_{2}^{12}+o\left(\tau_{1}^{6}+\tau_{2}^{12}\right)\right),
$$

$\tau \rightarrow(0,0)$, where $\operatorname{Re}\left(a \tau_{1}^{6}+b \tau_{1}^{4} \tau_{2}^{4}+d \tau_{2}^{12}\right)>0$ for $\tau \neq(0,0)$. (For details see [5, pp. 141-3].) Schreiber's result [9, p. 426] holds only for functions having about each absolute maximum a local expansion of the form

$$
\begin{aligned}
f\left(e^{i \tau}\right)= & c \exp \left(\beta \cdot \tau i-\sum_{j \geqq m_{1}} a_{j} \tau_{1}^{j}-\sum_{j \geqq m_{2}} b_{j} \tau_{2}^{j}-\sum_{j, k \geqq M} d_{j, k} \tau_{1}^{j} \tau_{2}^{k}\right. \\
& \left.+O\left(\|\tau\|^{M}\right)\right)
\end{aligned}
$$

$\tau \rightarrow(0,0)$, where $M=\max \left(m_{1}, m_{2}\right)$.

Define a polynomial $\rho$ in $n$ variables to be positive definite if $\rho(\tau)>0$ for all $\tau \neq \overrightarrow{0}$ and to be $q$-homogeneous of index $I$ if there exists an $n$-tuple $q$ of positive integers and a positive number $I$ such that for all $r>0$ and all $\sigma$ in $R^{n}, \rho\left(\left\langle r^{1 / q_{i}} \sigma_{i}\right\rangle\right)=r^{I} p(\sigma)$.

Let $T, Z, R$, and $C$ denote the unit circle, integers, real numbers and complex numbers respectively. For any set $S$ let $S^{n}$ denote the $n$-fold cartesian product of the set with itself. For any subset $S$ of $T^{n}$ let $C S$ denote the complement of $S$ in $T^{n}$. The letters $\sigma, \tau$, and $z$ denote points of $R^{n}, R^{n}$, and $C^{n}$ respectively. Alternatively denote a point $z$ of $C^{n}$ by using vector notation $\left\langle z_{i}\right\rangle$, where $z_{i}$ represents the $i$ th component of $z$, and let $e^{z}$ denote $\left\langle e^{z_{i}}\right\rangle$. Let $\overrightarrow{0}$ and $\overrightarrow{1}$ represent the origin of $R^{n}$ and the identity of $T^{n}$ respectively. The scalar product of two points $\sigma$ and $\tau$ of $R^{n}$ will be denoted $\sigma \cdot \tau$. Let $\delta_{i j}$ denote the Kronecker delta. Take products indexed by the empty set to be equal to one.

Let $B(\sigma, r)$ denote the set of points $e^{i \tau}$ of $T^{n}$ such that the Euclidean distance of $\tau$ from $\sigma$ is less than $r$. Let $E_{f}$ denote the set of points $e^{i \sigma}$ of $T^{n}$ such that $\left|f\left(e^{i \sigma}\right)\right|=1$. Let $L^{1}\left(R^{n}\right)$ and $A\left(R^{n}\right)$ 
denote the space of Lebesgue integrable functions on $R^{n}$ and the space of their Fourier transforms respectively. Similarly define $L^{1}\left(Z^{n}\right)$ and $A\left(T^{n}\right)$. For any function $\zeta$ on $T^{n}$ let supp $\zeta$ denote the support of $\zeta$.

Finally, the letters $a, b, c, d$ will denote absolute positive constants. The use of one such letter in two inequalities does not mean that the letter represents the same absolute constant in both inequalities. However, the use of such a letter in an inequality involving the indices $k$ or $m$ means that the constant represented by the letter is independent of $k$ and $m$. The phrases "for $k$ sufficiently large" and "for all $m$ " will be omitted finitely many times from this paper.

\section{Proof of theorem.}

LEMMA 1. Let $\rho$ be a positive definite, $q$-homogeneous polynomial of index 1. Then $q_{j}$ is an even integer for $1 \leqq j \leqq n$ and

$$
\sum_{j=1}^{n} \tau_{j}^{q} j<c_{n} \rho(\tau), \text { for all } \tau \neq 0
$$

Proof. Fix $j, 1 \leqq j \leqq n$. Let $h(t)=\rho\left(\left\langle t \delta_{i j}\right\rangle\right)$, for all $t$ in $R$. Since $\rho$ is $q$-homogeneous of index 1 ,

$$
h(t)=t^{q} h\left(\left\langle\delta_{i j}\right\rangle\right)
$$

for all $t>0$ and hence for all $t, h$ being a polynomial. Since $h$ is positive definite, $q_{j}$ is an even integer.

Assume that $\tau_{j} \neq 0$, let $r=\tau_{j}^{q j}$ and define $\sigma_{i}$ by $\tau=\left\langle r^{-1 / q_{i}} \sigma_{i}\right\rangle$. Since $\rho$ is $q$-homogeneous of index $1, \rho(\tau)=r \rho(\sigma)$. Since $\sigma_{j}=1$ and since $\rho$ is positive definite there is a positive constant $a_{j}$ such that $\rho(\sigma)>a_{j}$. Thus, $\rho(\tau) \geqq a_{j} \tau_{j}^{q}{ }$. Note that this inequality also holds if $\tau_{j}=0$. Summing over $j$ yields $n \rho(\tau) \geqq \sum_{j=1}^{n} a_{j} \tau_{j}^{q}$, from which the lemma follows since each $a_{j}$ is positive.

LemMa 2. Let $f$ be defined on $T^{n}$ and suppose that for some real number $r$ in the interval $(0, \pi) f$ vanishes on $C B(\sigma, r)$. Let $g$ be defined on $R^{n}$ by

$$
\begin{aligned}
& g(\tau)=f\left(e^{i(\sigma+\tau)}\right) \quad \text { if } \quad|\tau|<r, \\
& =0 \quad \text { if }|\tau| \geqq r .
\end{aligned}
$$

Then $f \in A\left(T^{n}\right)$ if and only if $g \in A\left(R^{n}\right)$. Moreover, there are positive numbers depending only on $r$ such that $a\|g\| \leqq\|f\|<b\|g\|$.

Proof. Without loss of generality, assume that $\sigma=0$. Then 
Lemma 2 is the special case of Lemma 7.7 of [9] obtained by letting $H=\{0\}$.

The following lemma is well-known and therefore its proof is omitted.

Lemma 3. Let $\lambda$ be a rotation of $R^{n}$ and $g$ be a function defined on $R^{n}$. For each $\mu \in L^{1}\left(R^{n}\right)$

$$
\widehat{\mu \circ \lambda}=\widehat{\mu} \circ \lambda
$$

so that $g \in A\left(R^{n}\right)$ if and only if $g \circ \lambda \in A\left(R^{n}\right)$. Moreover,

$$
\|g\|=\|g \circ \lambda\| \text {. }
$$

Lemma 4. There exists a constant $M=M(n)$ so that for any sequence $\left\{a_{q}\right\}_{q \in Z^{n}}$ of complex numbers and any constant $b$ in $C^{n}$

$$
\Sigma\left|a_{u}\right| \leqq M\left(\pi \Sigma \nu(q, l)\left|a_{q}\right|^{2}\right)^{2^{-n-1}}, n \geqq 1,
$$

where the sums are over all $q$ in $Z^{n}$, the product is over all $l \in$ $\Delta^{n} \equiv\{0,1\}^{n}$ and

$$
\nu(q, l)=\prod_{j=1}^{n}\left(1+\left|q_{j}-b_{j}\right|^{2}\right)^{1_{j}} .
$$

Proof. See Lemma 3 of [6].

LEMMA 5. Let $u$ and $w$ be q-homogeneous polynomials with indices $I_{u}$ and $I_{w}$ respectively. Let $\sigma$ be a point such that $u \cdot w(\sigma) \neq$ 0 . Then

$$
|u / w|\left(\left\langle r^{1 / q_{i}} \sigma_{i}\right\rangle\right)=0(1), \text { as } r \longrightarrow 0,
$$

if and only if $I_{u}>I_{w}$.

Proof. Applying the definition of $q$-homogeneity to $u$ and to $w$ yields

$$
|u / w|\left(\left\langle r^{1 / q_{i}} \sigma_{i}\right\rangle\right)=r^{I_{u}-I_{w}} u(\sigma) / w(\sigma)
$$

from which the lemma follows directly.

Lemma 6. Let u be a q-homogeneous, positive definite polynomial of index $I_{u}$ and $v$ a polynomial in $n$ variables such that

$$
|v(\tau)|=0(|u(\tau)|), \tau \longrightarrow \overrightarrow{0} .
$$

Then the index of q-homogeneity of each term of $v$ is greater than 
or equal to $I_{u}$.

Proof. It has been implicitly assumed in the statement of the lemma that each term of $v$ is $q$-homogeneous. It is straightforward to prove that in fact each term of any polynomial is $q$-homogeneous and that a sum of terms having a given index of $q$-homogeneity is $q$-homogeneous with that same index. Therefore, if $w$ is defined to be the sum of those terms of $v$ having smallest index of $q$-homogeneity and if $I_{w}$ denotes the index of $w$, it suffices to prove that $I_{w} \geqq I_{u}$.

Fix a point $\sigma$ such that $w(\sigma) \neq 0$. Since $w$ is $q$-homogeneous, $\sigma \neq \overrightarrow{0}$. From (7) it follows that

$$
|v / u|\left(\left\langle r^{1 / q_{i}} \sigma_{i}\right\rangle\right)=0(1), \text { as } r \longrightarrow 0 \text {. }
$$

Since $v-w$ is a sum of terms each of index exceeding $I_{v}$ and since $w(\sigma) \neq 0$ it follows that each term of $v-w$ upon division by $w$ and evaluation at $\left\langle\boldsymbol{r}^{1 / q_{i}} \sigma_{i}\right\rangle$ is either zero or by Lemma 5 approaches 0 as $r \rightarrow 0$. Thus,

$$
\left|\frac{v-w}{w}\right|\left(\left\langle r^{1 / q_{i}} \sigma_{i}\right\rangle\right)=o(1), \text { as } r \longrightarrow 0 .
$$

Since

$$
\frac{w}{u}=\frac{v}{u}(1+(v-w) / w)^{-1}
$$

it follows from (8) and (9) that

$$
\left|\frac{w}{u}\right|\left(\left\langle\boldsymbol{r}^{1 / q_{i}} \sigma_{i}\right\rangle\right)=0(1), \text { as } r \longrightarrow 0,
$$

which by Lemma 5 implies that $I_{u} \leqq I_{w}$ since $w(\sigma) \neq 0$ and $u(\sigma) \neq 0$, $u$ being positive definite.

LEMMA 7. Given any finite, pairwise-disjoint collection $\left\{B\left(\sigma, 2 r_{\sigma}\right)\right\}_{\sigma \in S}$ of open balls in $T^{n}$ there exists a family $\left\{\zeta_{\sigma}\right\}_{\sigma \in S}$ of nonnegative functions defined on $T^{n}$ such that for each $\sigma \in S$

(i) $\operatorname{supp} \zeta_{\sigma}=\overline{B\left(\sigma, 2 r_{\sigma}\right)}$,

(ii) $\zeta_{\sigma} \equiv 1$ on $B(\sigma, r)$,

(iii) $\zeta_{\sigma}\left(e^{i \tau}\right) \in C^{\infty}\left(R^{n}\right)$

and such that

(iv) $\sum_{\sigma \in S} \zeta_{\sigma} \leqq 1$ on $T^{n}$.

Proof. This lemma follows directly from Proposition 29 on page 254 of [3]. 
LEMMA 8. Let $f$ be defined on $T^{n}$, have an absolutely convergent Fourier series and satisfy (1). Further assume that $E_{f}$ is nonempty and finite. If $\left\{r_{\sigma}\right\}_{\sigma \in E_{f}}$ and $\left\{\zeta_{\sigma}\right\}_{\sigma \in E_{f}}$ satisfy (i) - (iv) of Lemma 7 and if, for each $\sigma \in E_{f}, \lambda_{\sigma}$ is a rotation of $R^{n}$ followed by translation by $\sigma$, then

$$
\begin{gathered}
\left\|f^{k}\right\|=0(1), k \longrightarrow \infty, \text { if }\left\|\left(\zeta_{\sigma} \cdot f^{k}\right) \circ e^{i \lambda_{\sigma}}\right\|=0(1), k \longrightarrow \infty, \\
\text { for each } \sigma \in E_{f} .
\end{gathered}
$$

Proof. This lemma is a direct consequence of Lemmas 2 and 3 of this paper and Theorem 3.3 of [9].

Proof of theorem. From (2), (3), and Lemma 1 it follows that for each point $e^{i \sigma}$ of the set $E_{f}$, which was defined in $\S 2$,

$$
\left|f\left(e^{i(\sigma+\lambda(\tau))}\right)\right| \leqq \exp \left(-c \sum_{j=1}^{n} \tau_{j}^{p_{j}}\right)
$$

for all $\tau$ in some neighborhood of $\overrightarrow{0}$ and hence that $E_{f}$ has no limit points. Since $T^{n}$ is compact, $E_{f}$ is finite. If $E_{f}=\varnothing$, then by the spectral radius formula $\left\|f^{k}\right\| \rightarrow 0$, as $k \rightarrow \infty$. So assume that $E_{f} \neq \varnothing$. Also, by Lemma 8 it suffices to assume that $E_{f}=\{\overrightarrow{0}\}$ and that $\lambda$ is the identity. Thus, it suffices to prove that

$$
\sum_{q \in Z^{n}}\left|a_{q, k}\right|=0(1), k \longrightarrow \infty,
$$

where the coefficients $a_{q, k}$ are defined by

$$
f^{k}\left(e^{i \tau}\right)=\sum_{q \in Z^{n}} a_{q, k} e^{i \tau \cdot q} .
$$

For this purpose let $b=k \beta$ and $a_{q}=a_{q, k}$, where $\beta$ is defined by (3), and show that the right side of (6) remains bounded, as $k$ tends to $\infty$, by showing for each $l$ in $\Delta^{n}$ that

$$
\sum_{q \in Z^{n}} \nu(q, l)\left|a_{q, k}\right|^{2}=0\left(k^{\left(2 \sum_{j \in J}-\sum_{j=1}^{n}\right)\left(1 / p_{j}\right)}\right),
$$

as $k \rightarrow \infty$, where $J$ denotes the set of integers $j$ for which $l_{j} \neq 0$.

Choose $r_{0}>0$ so that (3) and (10) hold for all $\tau$ such that $|\tau|<$ $r_{0}$. Let $B$ denote the set $\left\{\sigma \in R^{n}: e^{i \sigma} \in B\left(1, r_{0}\right)\right\}$. Let $\mathscr{D}$ denote the differential operator

$$
\prod_{j \in J}\left(-i \frac{\partial}{\partial t_{j}}-b_{j}\right)
$$

Recall that products indexed by the empty set are taken to be 1 so that if $J=\varnothing, \mathscr{D}$ is taken to be the identity. Use Parseval's 
equality to write

$$
c \sum_{q \in Z^{n}}\left(\prod_{j \in J}\left|q_{j}-b_{j}\right|^{2}\right)\left|a_{q, k}\right|^{2}=\int_{T^{n}}\left|\mathscr{D} f^{k}\left(e^{i \tau}\right)\right|^{2} d \tau
$$

and conclude that (11) will follow from

$$
\int_{B}\left|f^{k}\left(e^{i \tau}\right)\right|^{2} d \tau=0\left(k^{\left(2 \sum_{j \in J}-\sum_{j=1}^{n}\right)\left(1 / p_{j}\right)}\right)
$$

$k \rightarrow \infty$, and

$$
\int_{r^{n \backslash B}}\left|f^{k}\left(e^{i \tau}\right)\right|^{2} d \tau=0\left(e^{-c k}\right), k \rightarrow \infty .
$$

The last estimate is easy since for some $\delta$ in $(0,1),\left|f\left(e^{i \tau}\right)\right|<\delta$ on $T^{n} \backslash B$. Thus, to prove the theorem it suffices to establish the estimate given in (12). Since, by (3),

$$
f^{k}\left(e^{i \tau}\right)=c \exp k(\beta \cdot \tau i-(\rho+\gamma)(\tau))
$$

for all $\tau \in B$ and since $b=k \beta$ it follows that

$$
\left|\mathscr{D} f^{k}\left(e^{i \tau}\right)\right|=\left|\mathscr{D}_{J} \exp k(-\rho-\gamma)(\tau)\right|,
$$

for all $\tau \in B$, where for any subset $S$ of $\{1,2, \cdots, n\}$ the differential operator $\mathscr{D}_{S}$ is defined by $\mathscr{D}_{S}=\prod_{j \in S} \partial \mid \partial t_{j}$.

Expanding the expression on the right side of (13) by applying $\mathscr{D}_{J}$ yields

$$
\left|f^{k}\left(e^{i \tau}\right)\right|=\left|\sum_{\mathscr{J}} k^{|\curvearrowright|} \prod_{H \in \mathscr{U}}\left(-\mathscr{D}_{H}(\rho+\gamma)(\tau)\right) \exp k(-\rho-\gamma)(\tau)\right|,
$$

where the sum is over all partitions $\mathscr{P}$ of $J,|\mathscr{P}|$ denotes the number of members in $\mathscr{P}$ and the product is over all subsets $H$ of $J$ belonging to $\mathscr{P}$.

By hypothesis $\gamma \in C^{m}\left(R^{n}\right)$ where $m=\max _{1 \leqq i \leqq n} p_{i}$. Applying Taylor's formula yields

$$
\gamma(\tau)=v(\tau)+0\left(|\tau|^{m}\right), \tau \longrightarrow \overrightarrow{0},
$$

where $v(\tau)$ is a polynomial in $n$ variables. Since $|\gamma(\tau)|$ and $|\tau|^{m}$ are both $0\left(\sum \tau_{i}^{p_{i}}\right), \tau \rightarrow \overrightarrow{0}$, it follows from (15) that $|v(\tau)|$ is also. This implies by Lemma 6 that the index of $p$-homogeneity of each term of $v(\tau)$ is at least 1 since $\sum \tau_{i}^{p_{i}}$ is a $p$-homogeneous polynomial with index of $p$-homogeneity equal to 1 and since by Lemma $1 \sum \tau_{i}^{p_{i}}$ is positive definite. Thus, the polynomial $\mu \equiv \rho+v$ has no term with index of $p$-homogeneity less than 1 and

$$
(\rho+\gamma)(\tau)=\mu(\tau)+0\left(|\tau|^{m}\right), \tau \longrightarrow \overrightarrow{0} .
$$

Since $\rho, \gamma, \mu \in C^{m}\left(R^{n}\right), m \geqq n+1$, Taylor's formula and the last 
equality imply that

$$
\mathscr{D}_{H}(\rho+\gamma)(\tau)=\mathscr{D}_{H} \mu(\tau)+0\left(|\tau|^{m-|H|}\right),
$$

$\tau \rightarrow \overrightarrow{0}$, where $|H|$ denotes the number of elements in $H$. Since (3) and (10) hold for all $\tau \in B$,

$$
|\exp (-\rho-\gamma)(\tau)| \leqq \exp \left(-c \sum \tau_{j}^{p} j\right) .
$$

This, (14) and the preceding estimate imply that

$$
\left|\mathscr{D} f^{k}\left(e^{i \tau}\right)\right| \leqq \sum_{\mathscr{S}} k^{|\mathscr{P}|} \prod_{H \in \mathscr{S}}\left(\left|\mathscr{D}_{H} \mu(\tau)\right|+c|\tau|^{m-|H|}\right) \exp \left(-c \sum \tau_{j}^{p j}\right)
$$

where the sum and product are indexed as in (14).

For any $n$-tuple $q$ of nonnegative integers,

$$
\begin{aligned}
\mathscr{D}_{H}\left(\prod_{j} \tau_{j}^{q}\right) & =0 \text { if } q_{j}=0 \text { for some } j \text { in } H, \\
& =\prod_{j} \tau_{j}^{r} \text { otherwise },
\end{aligned}
$$

where

$$
\begin{aligned}
r_{j} & =q_{j}-1 \text { if } j \in H \\
& =q_{j} \text { otherwise } .
\end{aligned}
$$

Also, the index of $p$-homogeneity of any term $a \Pi_{j} \tau_{j}^{q}$ of $\mu$ is at least 1 , so that

$$
1 \leqq \sum q_{j} / p_{j}=\sum r_{j} / p_{j}+\sum_{j \in H} 1 / p_{j}
$$

Thus,

$$
\left|\mathscr{D}_{H} \mu\left(\left\langle k^{-1 / p_{j}} \tau_{j}\right\rangle\right)\right| \leqq c k^{-1+} \sum_{j \in H} \sum^{1 / p_{j}}
$$

for all $\tau \in B$. Also, since $m \geqq p_{j}$ for $1 \leqq j \leqq n$,

$$
\begin{aligned}
\left|\left\langle k^{-1 / p_{j}} \tau_{j}\right\rangle\right|^{m-|H|} & \leqq\left|\left\langle k^{-1 / m} \tau_{j}\right\rangle\right|^{m-|H|} \\
& \leqq c k^{-1+|H| / m} \\
& \leqq c k^{-1+} \sum_{j \in H} \sum^{1 / p_{j}}
\end{aligned}
$$

This estimate, (18) and (16) imply upon substitution of $k^{-1 / p_{j}} \tau_{j}$ for $\tau_{j}$ in (12) that

$$
\int_{B}\left|\mathscr{D} f^{k}\left(e^{i \tau}\right)\right|^{2} d \tau=0\left(\left(\sum_{\mathscr{\mathscr { N }}} k^{|\mathscr{O}|} \prod_{H \in \mathscr{O}} k^{\left.\left.-1+\sum_{j \in H}^{n} \sum^{1 / p_{j}}\right)^{2} k^{-} \sum_{j=1}^{1 / p_{j}}\right)},\right.\right.
$$

as $k \rightarrow \infty$, where the sum and product are indexed as in (14). From this estimate (12) follows and the theorem is proved. 


\section{REFERENCES}

1. B. Bajsanski, Sur une classe générale de procédés de Sommations du type d'EulerBorel, Acad. Serbe Sci. Publ. Inst. Math., 10 (1956), 131-152.

2. J. Clunie and P. Vermes, Regular Sonnenschein type summability methods, Acad. Roy. Belg. Bull. Cl. Sci., 45 (1959), 930-954.

3. W. Fleming, Functions of Several Variables, Addison-Wesley, Reading, MA, 1965.

4. G. W. Hedstrom, Norms of powers of absolutely convergent Fourier series in several variables, Michigan Math. J., 14 (1967), 493-495.

5. C. Heiberg, Norms of powers of absolutely convergent Fourier series: an example, Pacific J. Math., 66 (1976), 131-152.

6. - Norms of powers of absolutely convergent Fourier series of several variables, J. Functional Analysis, 14 (1973), 382-400.

7. J. P. Kahane, Algebras de convolucion de sucesiones, funciones y medidas sumables, in "Cursos y Seminarios de Mathematica", Vol. 6, Universidad de Buenos Aires, 1961.

8. D. J. Newman, Homomorphisms of $1_{+}$, Amer. J. Math., 91 (1969), 37-46.

9. B. M. Schreiber, Measures with bounded convolution powers, Trans. Amer. Math. Soc., 151 (1970), 405-431.

Received August 2, 1977 and in revised from March 30, 1978. This paper develops an idea introduced in the author's doctoral dissertation which was written at The Ohio State University and partially supported by NSF Grant \# GP 8959. The Naval Academy Research Council partially supported the writing of this paper and its presentation to the 82nd summer meeting of the Amer. Math. Soc. at Brown University on August 11, 1978.

United States Naval Academy

ANNAPOLIS, MD 21402 



\section{PACIFIC JOURNAL OF MATHEMATICS}

\section{EDITORS}

RICHARD ARENS (Managing Editor)

University of California

Los Angeles, California 90024

C. W. CURTIS

University of Oregon

Eugene, OR 97403

C. C. MOORE

University of California

Berkeley, CA 94720
J. DugundjI

Department of Mathematics University of Southern Californı Los Angeles, California 90007

R. Finn and J. Milgram Stanford University Stanford, California 94305

ASSOCIATE EDITORS

E. F. BECKENBACH

B. H. NeUmanN

F. WOLF

K. YoSHIDA

\section{SUPPORTING INSTITUTIONS}

UNIVERSITY OF BRITISH COLUMBIA CALIFORNIA INSTITUTE OF TECHNOLOGY

UNIVERSITY OF CALIFORNIA

MONTANA STATE UNIVERSITY

UNIVERSITY OF NEVADA, RENO

NEW MEXICO STATE UNIVERSITY

OREGON STATE UNIVERSITY

UNIVERSITY OF OREGON
UNIVERSITY OF SOUTHERN CALIFORNIA

STANFORD UNIVERSITY

UNIVERSITY OF HAWAII

UNIVERSITY OF TOKYO

UNIVERSITY OF UTAH

WASHINGTON STATE UNIVERSITY

UNIVERSITY OF WASHINGTON 


\section{Pacific Journal of Mathematics}

\section{Vol. 80, No. $1 \quad$ September, 1979}

Jeroen Bruijning and Jun-iti Nagata, A characterization of covering dimension by

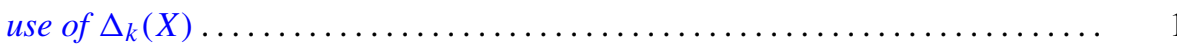

John J. Buoni and Albert Jonathan Klein, On the generalized Calkin algebra ...... 9

Thomas Ashland Chapman, Homotopy conditions which detect simple homotopy

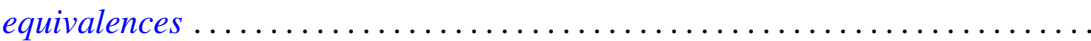

John Albert Chatfield, Solution for an integral equation with continuous interval

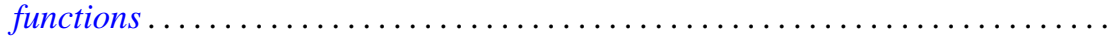

Ajit Kaur Chilana and Ajay Kumar, Spectral synthesis in Segal algebras on

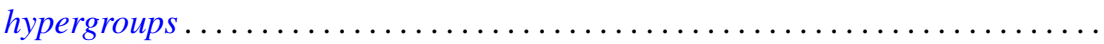

Lung O. Chung, Jiang Luh and Anthony N. Richoux, Derivations and

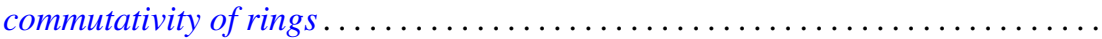

Michael George Cowling and Paul Rodway, Restrictions of certain function spaces to closed subgroups of locally compact groups .....................

David Dixon, The fundamental divisor of normal double points of surfaces........

Hans Georg Feichtinger, Colin C. Graham and Eric Howard Lakien,

Nonfactorization in commutative, weakly selfadjoint Banach algebras . . . . . . .

Michael Freedman, Cancelling 1-handles and some topological imbeddings ....... .

Frank E., III Gerth, The Iwasawa invariant $\mu$ for quadratic fields . . . . . . . . . . . . . .

Maurice Gilmore, Three-dimensional open books constructed from the identity

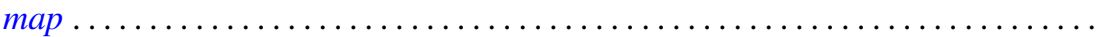

Stanley P. Gudder, A Radon-Nikodým theorem for $*$-algebras .

Peter Wamer Harley, III and George Frank McNulty, When is a point Borel? .

Charles Henry Heiberg, Fourier series with bounded convolution powers . .

Rebecca A. Herb, Characters of averaged discrete series on semisimple real Lie

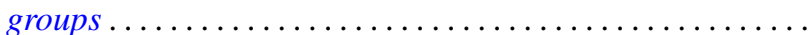

Hideo Imai, On singular indices of rotation free densities . .

Sushil Jajodia, On 2-dimensional CW-complexes with a single 2-cell . . .

Herbert Meyer Kamowitz, Compact operators of the form $u C_{\varphi}$

Matthew Liu and Billy E. Rhoades, Some properties of the Chebyshev method...

213

George Edgar Parker, Semigroups of continuous transformations and generating

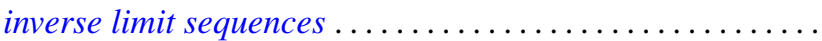

Samuel Murray Rankin, III, Oscillation results for a nonhomogeneous

equation ...

Martin Scharlemann, Transverse Whitehead triangulations ...

Gary Joseph Sherman, A lower bound for the number of conjugacy classes in a

finite nilpotent group

Richard Arthur Shoop, The Lebesgue constants for $\left(f, d_{n}\right)$-summability .

Stuart Jay Sidney, Functions which operate on the real part of a uniform

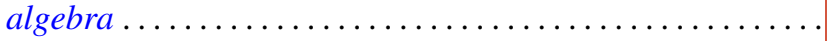

Tim Eden Traynor, The group-valued Lebesgue decomposition 